\title{
Identification of temperature field in thermoacoustic generator
}

\author{
Martin Veselýl,a, Petra Dančová ${ }^{1,2}$, Tomáš Vít $^{1}$, Vít Lédl ${ }^{1}$ \\ ${ }^{1}$ Technical University of Liberec, Studentská 2, Liberec 461 17, Czech Republic \\ ${ }^{2}$ Institute of Thermomechanics, CAS, v.v.i., Dolejškova 5, 18000 Praha 8, Czech Republic
}

\begin{abstract}
Presented paper includes description of design and assemblage of an experimental device for measuring temperature field into thermoacoustic generator using holographic interferometry method. This paper introduces the process of measurement on this apparatus and processing and analysis results too.
\end{abstract}

\section{Introduction}

The interaction of heat and sound has been a subject of interest to acousticians since 1816, when Laplace [1] corrected Newton's first theoretical calculation of the speed of sound in air, [2]. Newton assumed that the acoustic expansions and compressions of the gas occurred isothermally, without any associated variations in the temperature of the gas. Laplace included the effects of the changes in gas temperature that accompany the adiabatic expansions and compressions of the sound wave and derived the correct result for the adiabatic sound speed that was $18 \%$ faster than Newton's isothermal result. These thermal effects, which accompany sound waves, are essential to the operation of thermoacoustic engines and refrigerators, [3].

The term "thermoacoustics" was introduced by Nikolaus Rott, who claimed that "its meaning is rather self-explanatory", [4]. In the literal sense, Rott's claim is entirely justified, since the field is concerned with transformations between thermal and acoustical energy.

A detailed theoretical analysis of standing wave systems, based on the linear acoustics model was performed by Swift [5], who also provided some examples of the early developments at Los Alamos National Laboratory. He also provided a detailed analysis of a practical standing-wave engine where $7000 \mathrm{~W}$ of thermal energy was converted to $630 \mathrm{~W}$ of acoustic power, [6].

\section{Theory of thermoacoustic generators and digital holographic interferometry}

Thermoacoustic generator has a simple design and it is also a reliable device, which uses interaction between heat and acoustics for energy conversion [5].

The advantage of simple design of thermoacoustic devices means no moving parts, like are shafts, bearings, etc. It is not necessary any special, expensive or dangerous component materials, refills or lubricants. From this reason, the thermoacoustic devices are nature friendly and cheaper for production, than other refrigerators or electric generators.

In view of possibility operation with low temperature gradient, waste heat from a lot of industrial and energetic processes is possible to utilize.

Between disadvantages of thermoacoustic devices belongs fact that currently the majority of thermoacoustic devices have a low efficiency. The reason is that thermoacoustic phenomena is currently still in research stage.

But it is possible to assume that efficiency will increase and thermoacoustic engines and prime movers start to be more often used in practical applications.

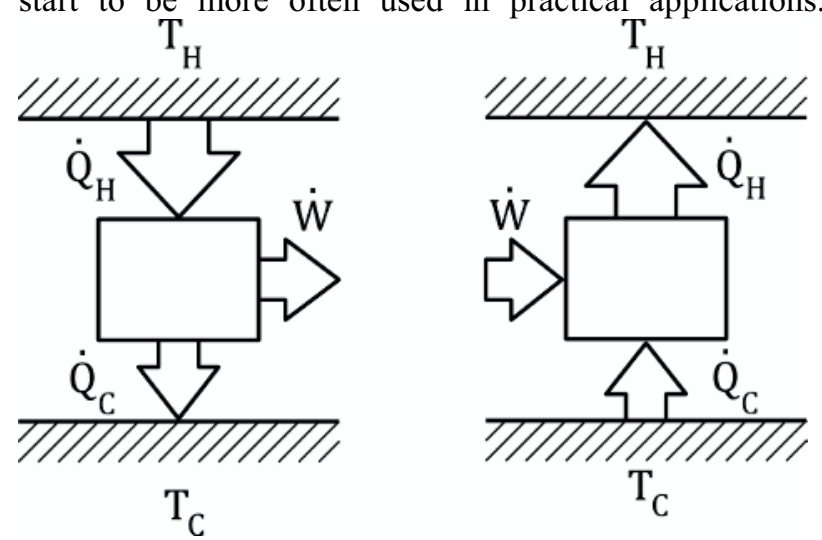

Figure 1. Schematic of function of thermoacoustic engine (left) and thermoacoustic heat pump (right)

\subsection{Schematic and description of a thermoacoustic device}

In thermoacoustic engines (figure 2), moves heat $\phi_{M}$ from a hot heat exchanger at a higher temperature $T_{X}$ to cold heat exchanger at a lower temperature $T_{\varepsilon}$ to produce

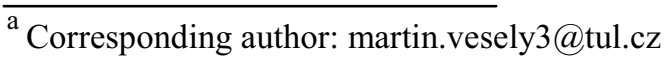


acoustic energy $W$ (which can be converted into another type of energy using, for example piezoelectricity), where is heat $\phi_{c}$ moved out.

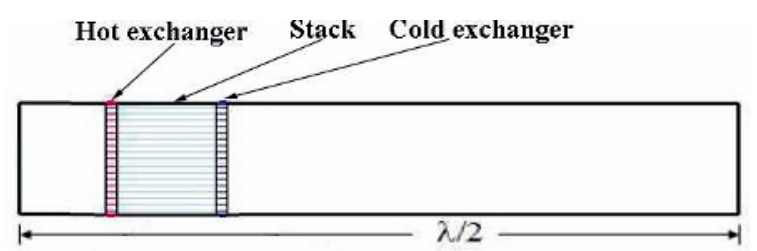

Figure 2. Schematic of thermoacoustic engine

In thermoacoustic heat pumps and refrigerators (figure 3 ), is acoustic energy $w$, that is produced for example by loudspeaker or by another thermoacoustic engine, used to pump heat $\phi_{c}$ from a cold heat exchanger at temperature $T_{F}$ to a hot heat exchanger at higher temperature $T_{H}$ where is obtained heat $\phi_{M}$.

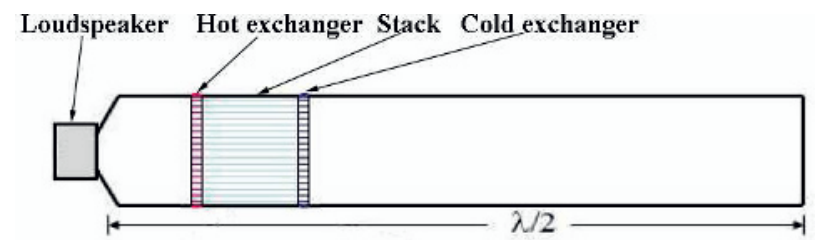

Figure 3. Schematic of thermoacoustic heat pump

It is useful that hot heat exchanger and cold heat exchanger create as low obstacles for flowing working gas inside resonator as is possible.

The most important part of any thermoacoustic device is stack, which significantly increases the efficiency of thermoacoustic devices. It is a ducts system between hot heat exchanger and cold heat exchanger inside resonator.

Ducts system can be made from parallel panes or from material with rectangular, square or hexagonal parallel holes. There can be used do not metal covered porous ceramic from car catalyst with good advantage.

Understanding the processes arising within the stack is the key for construction devices with the best possible efficiency.

\subsection{Temperature field measurement}

To measure the temperature field inside the stack, the digital holographic interferometry can be advantageously used.

Because it is an optical method, it is necessary to ensure the transparency of the model and produce a model of the material without significant optical defects.

To measure the temperature field with small temperature differences, an interferometer was designed and built at the Technical University in Liberec, [7], whose base is derived from Michaelson type. Mach Zehnder assembly does not achieve the required sensitivity. So light wave passes through the area measured twice. Interferograms are evaluated using custom software developed for this purpose.

The laser beam is split into two beams in the beam splitter (BS1), after the beam passes through the filter
(SF) is using the lens (CL) extended to the required width.

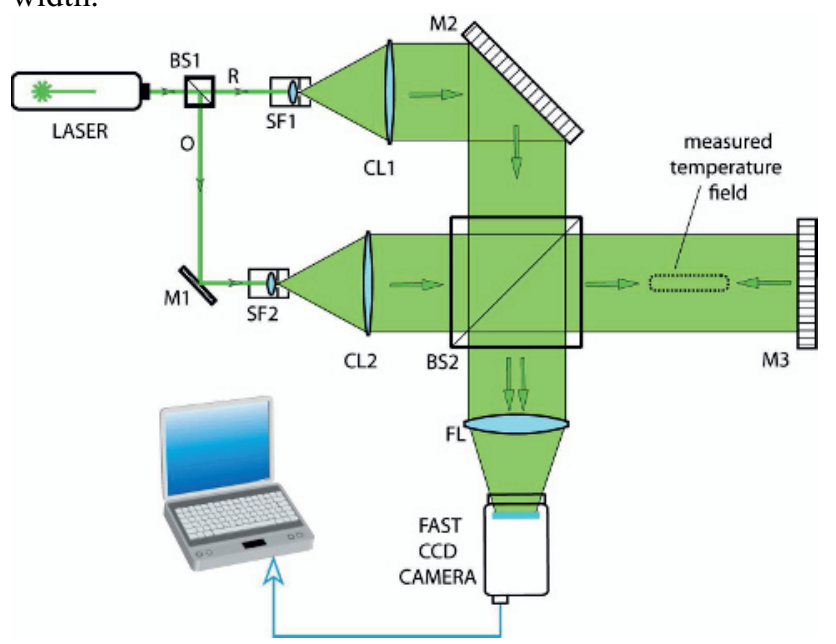

Figure 4. Schematic of digital holographic interferometer [7]

Collimated object beam $(\mathrm{O})$ enters the beam splitter (BS2), where one part is reflected and the other goes through the measured area and is normally incident on the mirror (M3), which is reflected and passes through the area measured again, which is a cause of increased sensitivity. Then again beam passes through splitter (BS2), wherein one part is reflected toward the CCD camera and a second part passes through.

The reference beam $(\mathrm{R})$ is directed along the collimation mirror (M2), to the beam splitter (BS2) through which a part goes together with a part the object beam carrying information from the measurement area. Both are then using the lens (FL) focused on the CCD where everyone falls under a slightly different angle.

\section{Device construction and measurement of temperature field}

\subsection{Device construction}

For device design a module for Microsoft Excel was programmed, which can calculate basic parameters from theoretical relationships.

In order to meet the requirement of transparency, the entire device is designed and manufactured from glass with the exception of heat exchangers, which are made of eloxed aluminum. Individual glass plates are glued together with a heat resistant silicone adhesive, which will also achieve a good seal.

Stack is made of thin parallel glass panes located between the hot and cold heat exchanger. Panes are glued into slots in the heat exchangers using heat-resistant inorganic adhesive.

All glass components are made from high temperature resistant silicious glasses that have small temperature extensibility and good temperature shock resistance. 


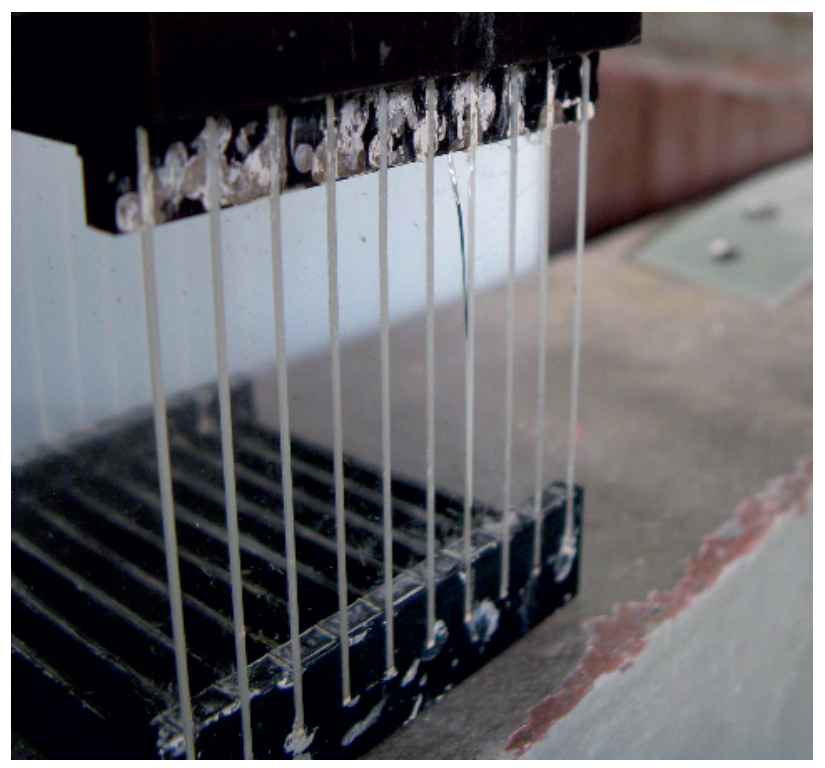

Figure 5. Detail of stack

Heater for heating of the hot exchanger uses electrical resistance. It is a resistance wire wrapped around the structure soldered of copper wire. Contact between copper construction and resistance wire is isolated using the same heat-resistant inorganic adhesive that was used for gluing stack.

Cooling of the cold exchanger is assisted with two Peltier elements located between inside and outside part of the cold exchanger. For better heat transfer from outside part of cold exchanger two fans are used.

At the end of the resonator the loudspeaker is mounted. The loudspeaker raises the resonant frequency of thermoacoustic process and allows device operation in heat pump mode.

The bottom part of thermoacoustic generator is shown on figure 5: electrical resistance heater (1), stack made from glass panes (2) that is lighted by laser beam and cold heat exchanger (3) that is composed from inside aluminum part, Peltier elements, outside aluminum heat sinks, fans and covering from paper, that are improve air flow through heat sinks and significantly improve cooling efficiency.

For reference temperature measurement the thermoacoustic generator is equipped with two thermocouples. First of them is mounted at side of hot heat exchanger, appropriately in middle. The second one is mounted at side of cold heat exchanger, analogous to first thermocouple.

\subsection{Measurement of temperature field}

The digital holographic interferometry was used for measurement of temperature field. Before the experiment can start, the first hologram had to be recorded. This hologram contains the data of the reference temperature distribution.

After thermoacoustic generator was turned on, another hologram was recoded. From reference hologram and actual hologram, it is possible to obtain information about distribution of temperature in temperature field.

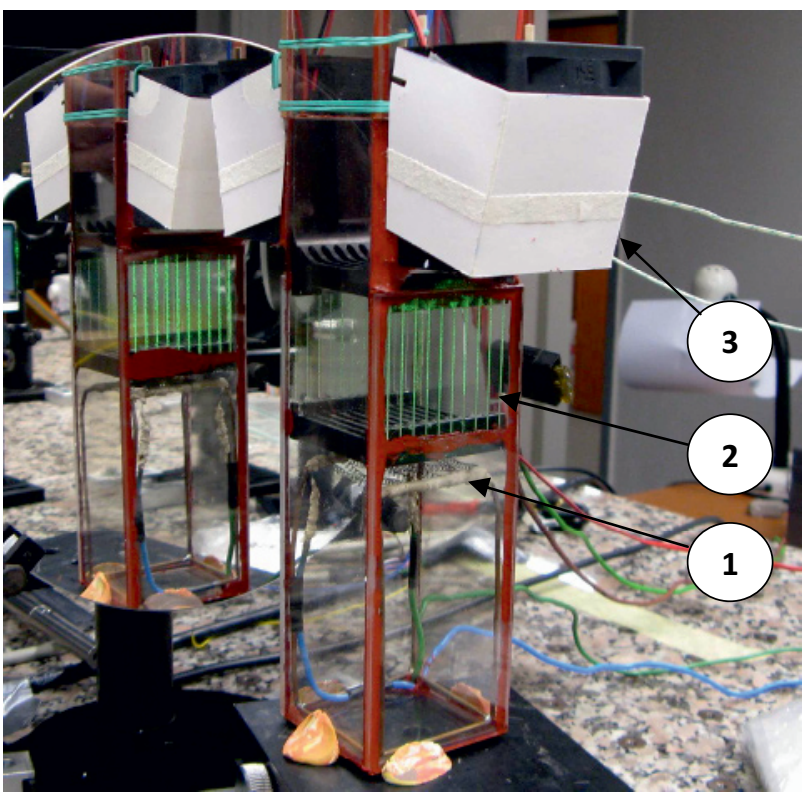

Figure 6. Thermoacoustic device inside digital holographic interferometer

For every hologram, the temperature values from both thermocouples were recorded. This data were used for evaluation of temperature fields obtained using holographic interferometry.

\section{Results}

\subsection{Measurement process and temperature values from thermocouples}

The first phase was carried out from measurements of temperature field without exciting loudspeaker. This was done for several temperature differences.

Table 1. Measuremet without exciting.

\begin{tabular}{|r|r|r|r|}
\hline$\#$ & $\begin{array}{c}\text { Cold exchanger } \\
{\left[{ }^{\circ} \mathrm{C}\right]}\end{array}$ & $\begin{array}{c}\text { Hot exchanger } \\
{\left[{ }^{\circ} \mathrm{C}\right]}\end{array}$ & $\begin{array}{c}\text { Temperature } \\
\text { difference }\left[{ }^{\circ} \mathrm{C}\right]\end{array}$ \\
\hline 1 & 25,1 & 57,0 & 31,9 \\
\hline 2 & 25,4 & 65,8 & 40,4 \\
\hline 3 & 25,7 & 75,2 & 49,5 \\
\hline 4 & 26,2 & 85,8 & 59,6 \\
\hline 5 & 27,1 & 96,0 & 68,9 \\
\hline 6 & 28,1 & 105,5 & 77,4 \\
\hline
\end{tabular}

The main purpose of this measurement was to determine whether the digital holographic interferometer is set up and whether providing usable temperature field images.

In the second stage, measurements were taken with excitation of temperature field using loudspeaker. The heating power was $10 \mathrm{~W}, 15 \mathrm{~W}, 20 \mathrm{~W}$ and $25 \mathrm{~W}$. Sensing cameras was synchronized with the excitation and was always twelve images captured along the amplitude of excitation. Because of the camera was too slow, it could be not taken all images in single cycle. Hence images were captured in multiple cycles in defined stages. These stages are descripted at figure 7. 


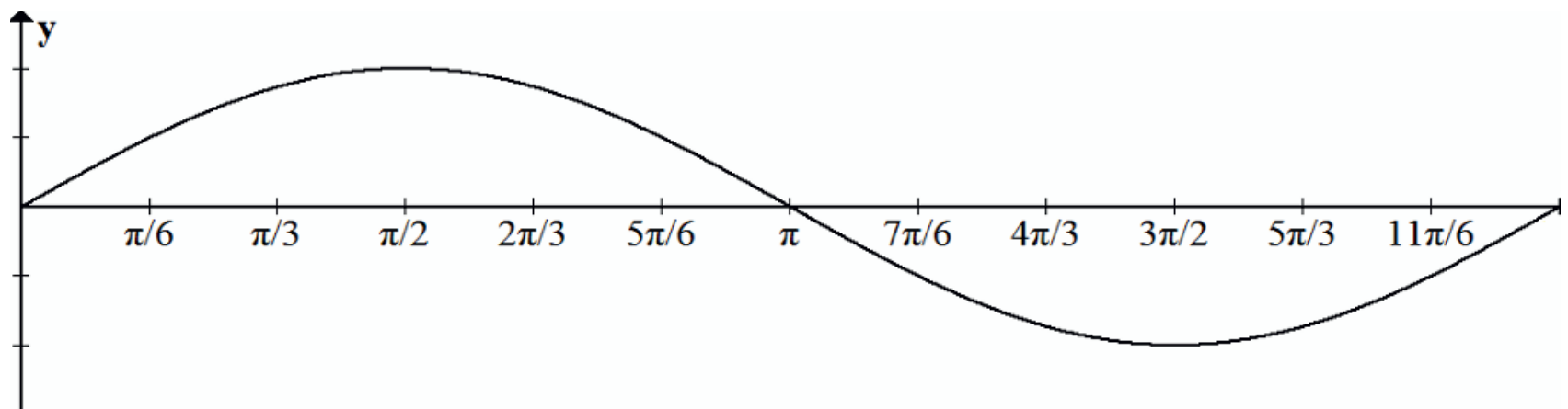

Figure 7. Image location along the acoustic wave

The measured values are placed in the following table:

Table 2. Measuremet with exciting.

\begin{tabular}{|c|c|c|c|c|}
\hline & \multicolumn{2}{|c|}{$10 \mathrm{~W}$} & \multicolumn{2}{|c|}{$15 \mathrm{~W}$} \\
\hline & $\mathrm{T}_{\mathrm{C}}\left[{ }^{\circ} \mathrm{C}\right]$ & $\mathrm{T}_{\mathrm{H}}\left[{ }^{\circ} \mathrm{C}\right]$ & $\mathrm{T}_{\mathrm{C}}\left[{ }^{\circ} \mathrm{C}\right]$ & $\mathrm{T}_{\mathrm{H}}\left[{ }^{\circ} \mathrm{C}\right]$ \\
\hline $0 \pi$ & 25,0 & 62,1 & 28,2 & 87,2 \\
\hline $1 / 6 \pi$ & 25,2 & 62,5 & 28,5 & 87,3 \\
\hline $2 / 6 \pi$ & 25,3 & 62,8 & 28,8 & 87,4 \\
\hline $3 / 6 \pi$ & 25,5 & 63,2 & 29,2 & 87,6 \\
\hline $4 / 6 \pi$ & 25,6 & 63,5 & 29,5 & 87,7 \\
\hline $5 / 6 \pi$ & 25,8 & 63,9 & 29,8 & 87,8 \\
\hline $6 / 6 \pi$ & 25,9 & 64,2 & 30,1 & 87,9 \\
\hline $7 / 6 \pi$ & 26,1 & 64,6 & 30,4 & 88,0 \\
\hline $8 / 6 \pi$ & 26,2 & 64,9 & 30,7 & 88,1 \\
\hline $9 / 6 \pi$ & 26,4 & 65,3 & 31,1 & 88,3 \\
\hline $10 / 6 \pi$ & 26,5 & 65,6 & 31,4 & 88,4 \\
\hline \multirow[t]{3}{*}{$11 / 6 \pi$} & 26,7 & 66,0 & 31,7 & 88,5 \\
\hline & \multicolumn{2}{|c|}{$20 \mathrm{~W}$} & \multicolumn{2}{|c|}{$25 \mathrm{~W}$} \\
\hline & $\mathrm{T}_{\mathrm{C}}\left[{ }^{\circ} \mathrm{C}\right]$ & $\mathrm{T}_{\mathrm{H}}\left[{ }^{\circ} \mathrm{C}\right]$ & $\mathrm{T}_{\mathrm{C}}\left[{ }^{\circ} \mathrm{C}\right]$ & $\mathrm{T}_{\mathrm{H}}\left[{ }^{\circ} \mathrm{C}\right]$ \\
\hline $0 \pi$ & 30,8 & 94,7 & 37,2 & 117,2 \\
\hline $1 / 6 \pi$ & 31,0 & 95,1 & 37,3 & 117,4 \\
\hline $2 / 6 \pi$ & 31,3 & 95,4 & 37,5 & 117,5 \\
\hline $3 / 6 \pi$ & 31,5 & 95,8 & 37,6 & 117,7 \\
\hline $4 / 6 \pi$ & 31,7 & 96,2 & 37,7 & 117,8 \\
\hline $5 / 6 \pi$ & 31,9 & 96,5 & 37,8 & 118,0 \\
\hline $6 / 6 \pi$ & 32,2 & 96,9 & 38,0 & 118,1 \\
\hline $7 / 6 \pi$ & 32,4 & 97,2 & 38,1 & 118,3 \\
\hline $8 / 6 \pi$ & 32,6 & 97,6 & 38,2 & 118,4 \\
\hline $9 / 6 \pi$ & 32,8 & 98,0 & 38,3 & 118,6 \\
\hline $10 / 6 \pi$ & 33,1 & 98,3 & 38,5 & 118,7 \\
\hline $11 / 6 \pi$ & 33,3 & 98,7 & 38,6 & 118,9 \\
\hline
\end{tabular}

\subsection{Evaluated temperature fields}

For example two images of temperature field measured without excitation. At figure 8 is shown evaluated temperature field of smallest temperature difference and at figure 9 is shown evaluated temperature field of highest temperature difference.

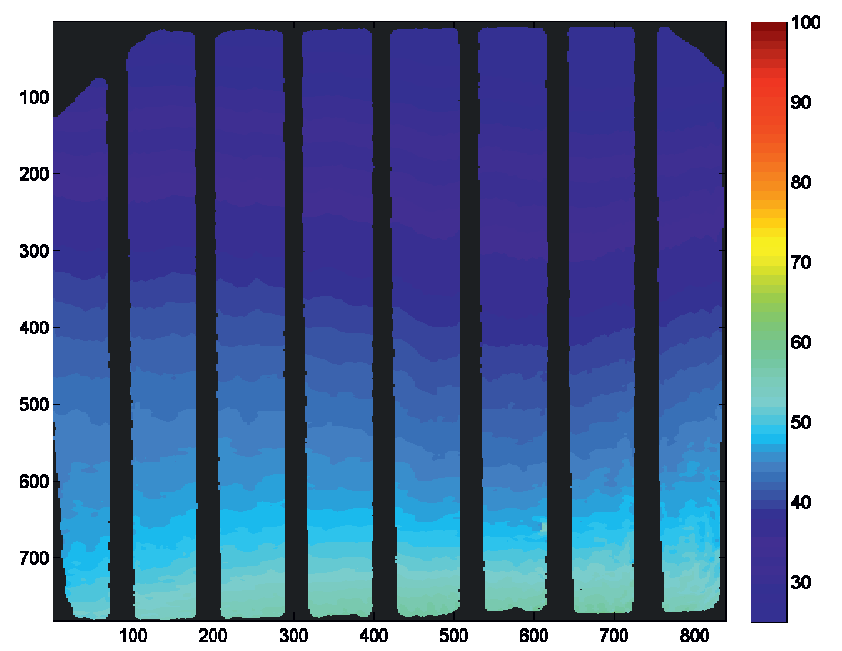

Figure 8. Temperature field without excitation (\#1)

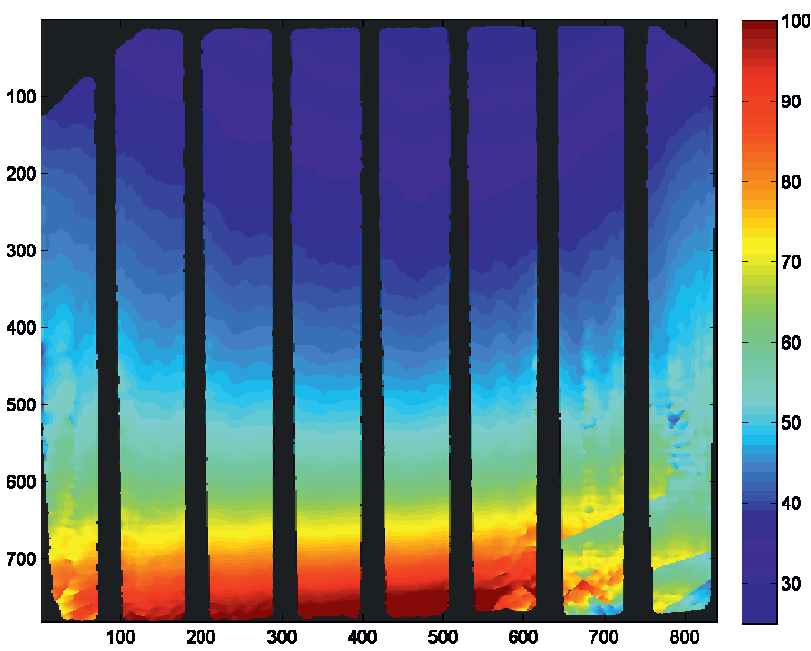

Figure 9. Temperature field without excitation (\#6)

On few following examples are evaluated temperature fields with excitation with loudspeaker and heater at $20 \mathrm{~W}$. Figure 10 shows the temperature field in first node of standing acoustic wave. At figure 11 is temperature field in distance $5 / 6 \pi$ from first node. It is nearby second node. At figure 12 is temperature field in distance $11 / 6 \pi$ from first node. 


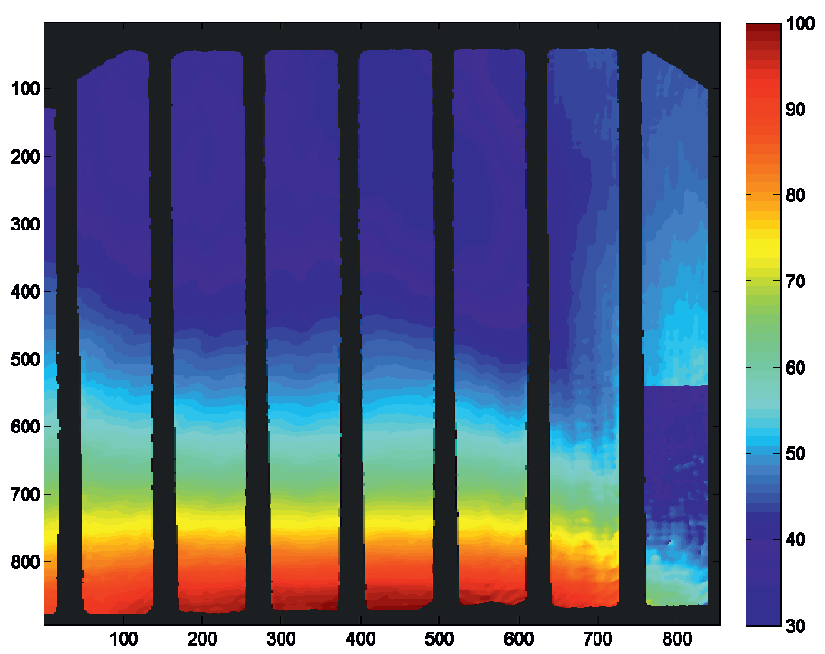

Figure 10. Temperature field with excitation $(0 \pi)$

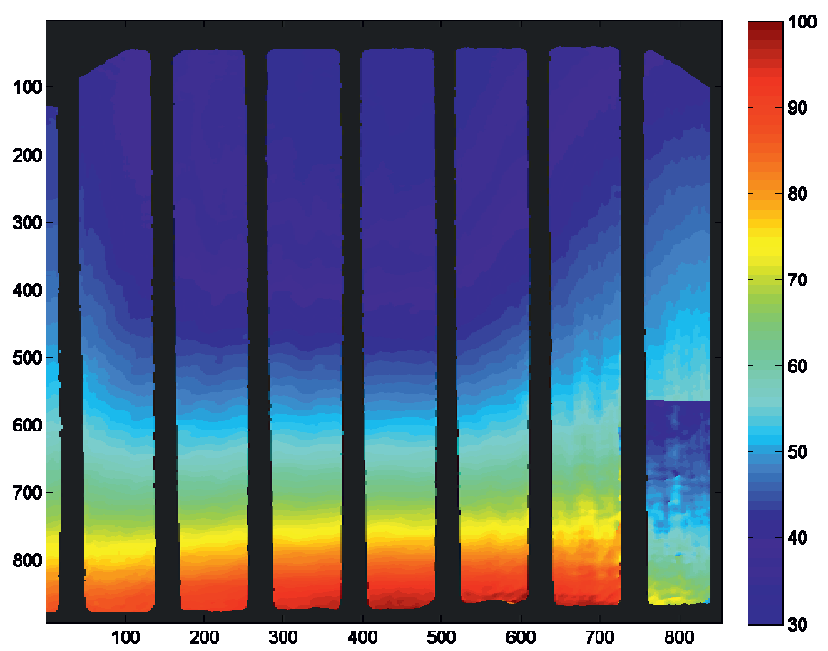

Figure 11. Temperature field with excitation $(5 / 6 \pi)$

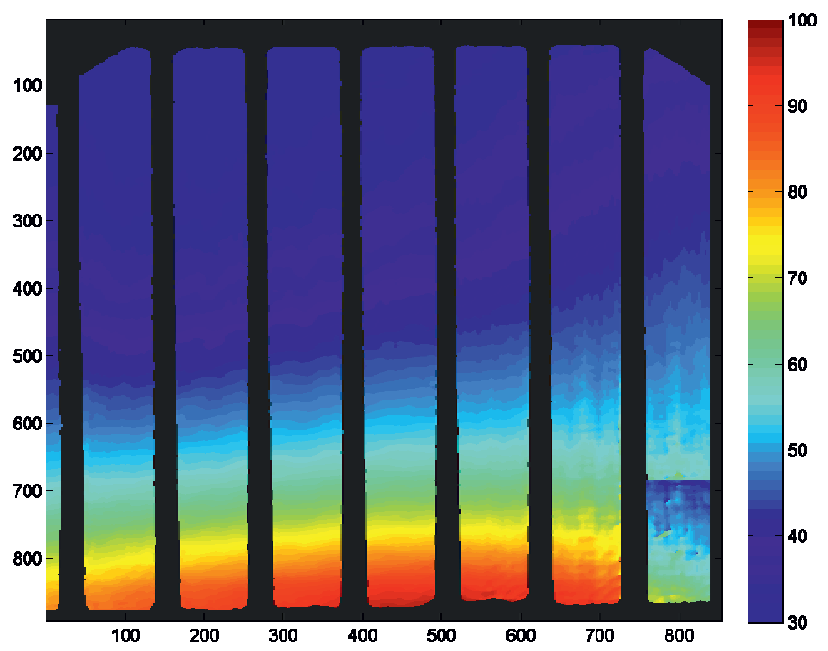

Figure 12. Temperature field with excitation $(11 / 6 \pi)$

All images obtained by holographic interferometry were evaluated and from every sequence images obtained at different heating power the video was created.

\section{Conclusions}

The main objective of this work was to investigate the behavior of the temperature field inside the stack in the course of thermoacoustic phenomenon.

This destination was not reached, because during the measurement was either failed to reach thermoacoustic phenomenon, or thermoacoustic phenomenon was so weak that he could not identify.

That does not change the number of lessons learned applicable to future research, whether it is the choice of construction materials as, or technological processes in the production of individual components.

For other measurements, it is possible to recommend several improvements.

It is highly recommended to add automatic temperature control for hot and cold heat exchanger. Also increase efficiency of device is needed. That can be made by reducing spaces between stack panes, but that spaces must be still so large for measurement temperature field using digital holographic interferometry. Also improving cooling efficiency can provide higher temperature at stack. The better efficiency can be also reached using another working gas inside device.

\section{Acknowledgements}

The authors would like to thank the Grant Agency of Czech Republic GACR (project no. P101/11/J019).

This work was supported by ESF operational programme "Education for Competitivness" in the Czech Republic in the framework of project „Support of engeneering of excellent research and development teams at the Technical University of Liberec" No. CZ.1.07/2.3.00/30.0065.

We also thank to project SGS 28000.

\section{References}

1. P. S. Laplace, Ann. Chim. Phys. 3, 238 (1816)

2. I. Newton, Principia Mathematica, Book II, Sec. VIII (1687)

3. N. Rott, Advances in Applied Mechanics 20, 13 (1980)

4. J. C. Wheatley, G. W. Swift, A. Migliori, "Acoustical heat pumping engine," US Pat. No. 4,398,398 (1983).

5. G. W. Swift, J Acoust Soc Am 84:1145(1988)

6. G. W. Swift, J Acoust Soc Am 92:1551(1992)

7. V. Lédl, T. Vít, R. Doleček, P. Psota: EPJ Web of Conferences 25 (02014) (2012) 